Revue européenne des sciences sociales

European Journal of Social Sciences

XLII-129 | 2004

La sociologie durkheimienne : tradition et actualité

\title{
Les naissances « au fil des saisons »
}

Arnaud Régnier-Loilier

\section{OpenEdition}

\section{Journals}

Édition électronique

URL : http://journals.openedition.org/ress/431

DOI : $10.4000 /$ ress.431

ISSN : 1663-4446

\section{Éditeur}

Librairie Droz

\section{Édition imprimée}

Date de publication : 1 mars 2004

Pagination : 293-305

ISBN : 2-600-00941-8

ISSN : 0048-8046

Référence électronique

Arnaud Régnier-Loilier, "Les naissances « au fil des saisons » ", Revue européenne des sciences sociales [En ligne], XLII-129 | 2004, mis en ligne le 06 novembre 2009, consulté le 19 avril 2019. URL: http://journals.openedition.org/ress/431; DOI : 10.4000/ress.431 
Arnaud RÉGNIER-LOILIER

\section{LES NAISSANCES «AU FIL DES SAISONS»}

\section{POSITION DU PROBLÈME ET PANORAMA HISTORIQUE}

En 1989, Philippe Besnard s'est intéressé aux «effets de la ronde annuelle des saisons sur la vie des Français, sur leurs comportements, leurs habitudes, leurs humeurs et leurs désirs ", sujet selon lui trop peu étudié et mal connu des sociologues. Dans un ouvrage dédié à l'étude des Mours et humeurs des Français au fil des saisons ${ }^{1}$, il passait alors en revue un certain nombre de phénomènes humains et sociaux portant en eux l'empreinte du rythme des saisons et cherchait à en proposer quelques pistes explicatives. Parmi les différents thèmes développés, l'auteur posait son regard sur «la saison des amours», en essayant de mieux comprendre la répartition infra-annuelle des naissances en France. Si au $\mathrm{XVII}^{\mathrm{e}}$ et $\mathrm{XVIII}{ }^{\mathrm{e}}$ siècles les accouchements étaient nettement plus nombreux au sortir de l'hiver (février, mars), les mois de juin, juillet et décembre étant les plus pauvres de l'année en naissances, le profil saisonnier s'est ensuite modifié au fil du temps, à la fois en perdant de son amplitude et en voyant son mode se déplacer progressivement vers le mois de mai, juillet lui ayant depuis quelques années volé la vedette. Cette évolution dans le temps rendrait ainsi fragile les interprétations de type naturaliste selon lesquelles l'homme aurait une reproduction saisonnière ${ }^{2}$.

En s'appuyant sur des travaux d'historiens et de démographes, Philippe Besnard rend compte de cette évolution par divers bouleversements sociaux. La forme du mouvement saisonnier des naissances dans la France ancienne a d'abord souvent été lue comme la conséquence du calendrier chrétien qui recommandait de s'abstenir de tout «commerce conjugal au moment des fêtes religieuses et durant les temps de pénitence: l'Avent et le Carême. Enfreindre ces interdits, rappelle le sociologue, était s'exposer au châtiment divin et au risque de mettre au monde des enfants monstrueux $»^{3}$. Les conceptions étaient alors moins

Paris, Balland.

2 Les premiers à s'intéresser au mouvement saisonnier des naissances - par exemple Louis-René Villermé dans son rapport Sur la distribution par mois des conceptions et des naissances de 1831 - l'ont d'abord interprété comme la conséquence de la variation des conditions climatiques au long de l'année. Le climat pouvait avoir, selon eux, une incidence sur les mécanismes physiologiques de la reproduction ou sur la fréquence des rapports sexuels.

3 Ph. Besnard, Mours et humeurs, p. 21. 
nombreuses en ces temps de l'année ${ }^{4}$. Mais, progressivement, les couples se sont détachés de ces préceptes religieux, entraînant du même coup un tassement des oscillations mensuelles. Jacques Houdaille a pour sa part suggéré l'existence d'un lien entre la répartition mensuelle des naissances et la vie des campagnes. Les grands travaux des champs de l'été entraînaient alors fatigue, indisponibilité voire même séparation entre conjoints pour de très nombreux travailleurs saisonniers qui louaient leurs bras durant les périodes de forte activités. Philippe Besnard propose également une explication rationnelle observant que «les naissances de l'hiver finissant paraissent assez fonctionnelles dans le calendrier paysan »: «on conçoit peu en novembre pour ne pas être indisponible en août ${ }^{\circ}$. Le déclin progressif de la part des paysans dans la population active permettrait de rendre compte de l'érosion constante de l'amplitude des variations saisonnières au fil des siècles. Le mouvement saisonnier s'est ensuite modifié à partir du XIX ${ }^{\mathrm{e}}$ siècle, le pic de février-mars laissant place à partir des années mille neuf cent cinquante à un surplus de naissances au printemps, centré sur le mois de mai, évolution souvent mise en regard avec l'instauration des congés payés en France en 1936. Les vacances seraient un moment privilégié pour les accouplements féconds, les conjoints étant plus que jamais disponibles et disposés aux ébats amoureux durant ce temps sacré de l'année ${ }^{7}$. Toutefois, en s'appuyant sur la distribution journalière des naissances, Philippe Besnard émet une réserve quant à cette piste explicative observant pour la période 1982-1988 que les vacances sont davantage «productives en conceptions par les rentrées qu'elles provoquent $»^{8}$.

Ainsi, pour la période ancienne, la plupart des explications proposées renvoient à des facteurs extérieurs qui modèleraient de manière indirecte la tendance saisonnière. En revanche, l'auteur suggère pour la période plus récente et en prolongement d'hypothèses proposées dans les années soixante-dix par certains démographes ${ }^{9}$, d'interpréter les fluctuations saisonnières contemporaines comme le résultat possible d'une planification des grossesses de la part des couples. Resterait alors à mettre à jour les motivations des conjoints à programmer si finement leur fécondité afin de comprendre la forme globale du mouvement saisonnier contemporain. Nous avons souhaité poursuivre cette investigation en mettant en place une étude spécifique à la question (voir encadré méthodologique).

4 Certains chercheurs ont avancé une hypothèse concurrente à celle-ci voyant dans les fluctuations saisonnières des conceptions non pas les conséquences de l'observance des préceptes religieux qui prônaient l'abstinence périodique mais un effet indirect des pratiques alimentaires durant ces temps de pénitence (jeûne du Carême) sur les mécanismes physiologiques de la reproduction.

5 Voir J. Houdaille, «Mouvement saisonnier des conceptions en France de 1740 à $1829 »$, Population, 1979, n. 2, pp. 452-457; «Le mouvement saisonnier des conception dans la France rurale de 1640 à $1669 »$, Population, 1985, n. 2, pp. 360-362.

$6 \quad$ Ph. Besnard, Mours et humeurs, p. 41.

7 Gérard Calot propose également cette explication suite à une étude comparative entre la France, l'Angleterre - Pays de Galles et la République Fédérale d'Allemagne. Voir G. Calot, «L'observation de la fécondité à court et moyen terme », Population, 1981, n. 1, pp. 9-40.

$8 \quad$ Voir Ph. Besnard, Mœurs et humeurs, p. 36.

9 Voir par exemple les conclusions proposées par Henri Leridon au terme de son ouvrage Natalité, saisons et conjoncture économique, Paris, INED-PUF, 1973, ou celles de Michel Dupâquier, Le mouvement saisonnier des naissances en France (1853-1973), thèse de doctorat, 1976. 


\section{Encadré méthodologique}

En 1999, en partenariat avec la Direction de la Population et des Migrations (DPM) et l'Institut National d'Études Démographiques (INED) ${ }^{10}$, nous avons mis en place une «Etude portant sur la période de naissance des enfants dans l'année». Cette enquête par questionnaires auto-administrés s'est déroulée sur douze mois, de novembre 1999 à octobre 2000 dans cinq maternités de la région Nord-Pas-de-Calais et a permis de recueillir le point de vue de 2.906 jeunes femmes venant d'accoucher ${ }^{11}$.

Parallèlement, quarante-cinq entretiens ont été réalisés auprès de jeunes parents attendant ou venant d'avoir un enfant, dans le but de mieux comprendre la manière dont la venue d'un enfant se planifie au cours du cycle de vie. On s'attachait notamment à observer l'existence ou non de stratégies saisonnières, à en comprendre les motivations et les enjeux pour les parents.

Les résultats (statistiques et extraits d'entretien) proposés dans le texte pour lesquels la source n'est pas précisée renvoient implicitement à ces deux études.

Au cours des pages qui suivent, nous souhaitons rendre compte de quelquesuns des résultats obtenus à la suite de l'exploitation des données recueillies ${ }^{12}$. L'hypothèse d'une planification des naissances dans l'année peut-elle être validée? Comment comprendre les motivations des parents à choisir de manière plus ou moins fine la période de naissance de leurs enfants dans l'année ? Malgré sa faible amplitude, le mouvement saisonnier des naissances peut-il être interprété comme la résultante des stratégies mises en place par les couples?

\section{LA PLANIFICATION DES NAISSANCES DANS L'ANNÉE: UNE RÉALITÉ}

En ce début de vingt et unième siècle, la pratique contraceptive est en France presque généralisée, principalement aux jeunes âges ${ }^{13}$. Les techniques utilisées sont majoritairement médicalisées (pilule, stérilet) et se caractérisent par leur «permanence» tout au long du cycle menstruel de la femme. Ainsi, le souhait d'avoir un enfant nécessite d'abord pour le couple de s'affranchir de l'usage de la contraception, impliquant de sa part une démarche volontaire, à un moment donné. La durée approximative d'une grossesse (neuf mois) étant connue de la plupart des conjoints, il apparaît pertinent de supposer que certains d'entre eux choisissent le moment de l'année auquel ils vont se défaire de leur protection

10 Merci à Henri Leridon (Inéd-Inserm) pour son soutien.

11 Pour plus de détails, voir A. Régnier-Loilier, Stratégies des couples en matière de fécondité. De la "fécondité naturelle» à l" «infécondabilité naturelle», thèse de doctorat sous la direction de J.-C. Rabier, Institut de sociologie, Université de Lille 1, décembre 2002 (Partie I, chapitre 3).

12 Les résultats présentés sont extraits d'un travail de recherche plus global portant sur les «stratégies des couples en matière de fécondité». Cf. A. Régnier-Loilier, ibid., 2002.

13 La protection contraceptive est de l'ordre de 97\%. Voir C. de Guibert- Lantoine, H. Leridon, «La contraception en France. Un bilan après trente ans de libéralisation », Population, 1998, n. 4, pp. 785-812. 
contraceptive dans le but d'avoir un enfant à une période particulière de l'année. Reste alors à appréhender leurs motivations, leurs «stratégies».

\section{a) L'attrait de la «belle saison»}

La plus fréquente des motivations a trait aux avantages d'avoir un enfant au moment des «beaux jours ». Les bénéfices sont doubles, à la fois partagés entre la jeune maman et son nourrisson. En premier lieu, la perspective d'une naissance au cœur de l'hiver n'enchante guère les futures mamans; elles resteraient confinées chez elles, les conditions climatiques se posant en obstacle aux promenades avec leur nouveau-né et risquant aussi de nuire à leur équilibre psychologique déjà perturbé par l'arrivée d'un nouveau membre dans la famille. Se développe par ailleurs un imaginaire autour de la santé de l'enfant, relativement à sa période de naissance dans l'année. Certaines femmes perçoivent en effet dans une naissance au deuxième trimestre des bienfaits pour leur nourrisson. Né loin de la saison froide et humide et du cortège des maladies hivernales, le bébé pourra bénéficier de la belle saison pour grandir, se renforcer et construire son système de défenses immunitaires. Il sera ainsi plus à même d'affronter le retour de la saison froide et mieux paré pour traverser l'hiver. Ces deux motifs - attrait des beaux jours et santé de l'enfant - contribuent tous deux à une répartition des naissances dans l'année qui se concentre nettement entre le mois de mars et le mois de juin, le mode se positionnant sur mai.

Si les deux arguments présentés plaident en faveur d'accouchements de printemps, les femmes mettent principalement en avant les gains directs qu'elles peuvent en retirer elles-mêmes pour justifier de leur programmation. Parmi celles qui ont déclaré avoir planifié la période dans l'année, $47 \%$ souhaitaient «avoir un enfant à la belle saison pour pouvoir sortir facilement» alors que seulement $15 \%$ estimaient qu'il était préférable «pour la santé de l'enfant» qu'il naisse à une autre saison que l'automne ou l'hiver. Ce sont principalement les femmes des classes moyennes et supérieures («professions intermédiaires» et, dans une moindre mesure, «cadres et professions intellectuelles supérieures») qui attachent de l'importance à la raison «santé de l'enfant». Elles se recrutent plus particulièrement parmi les «professions intermédiaires de la santé et du travail social», peut-être en raison de l'intérêt qu'elles portent quotidiennement aux questions de santé.

\section{b) Le cumul du congé de maternité et des vacances d'été}

Le désir de souder le congé de maternité aux vacances d'été alimente également la saison du printemps en naissances. Une femme sur quatre ayant programmé la venue de son enfant dans l'année poursuivait cette finalité, dans un cas sur deux parce qu'elle n'avait pas la possibilité de choisir ses dates de vacances. L'enjeu est d'éviter que le congé de maternité vienne se superposer aux congés professionnels le plus souvent fixés sur les mois de juillet et août (87\% et $97 \%$ respectivement), ce qui occasionnerait une «perte» de jours potentiels d'inactivité. «C'est ça que je ne voulais pas, c'est perdre des congés parce que ça tombe juste avant les vacances ou pendant les vacances (...). Parce que ceux qui sont dans le privé, effectivement ils peuvent rajouter leurs congés après le congé de maternité. Moi, c'est fixé d'avance donc je ne peux pas faire ça». Les 
meilleures représentantes de ce schéma stratégique sont les femmes institutrices et, dans une moindre mesure, professeurs de lycée ou enseignantes à l'université. «Etant enseignante, ça m'arrangeait bien d'enchaîner mon congé de maternité avec les deux mois de grandes vacances (...). [Avril], c' est vraiment le meilleur mois parce que je ne reprends pas et j'enchaîne avec les grandes vacances». Compte tenu de la durée du congé de maternité auquel elles peuvent prétendre, variable selon le nombre d'enfants qu'elles ont déjà, elles essayent alors de faire coïncider la fin de celui-ci avec le début des grandes vacances scolaires. Ceci entraîne un net déficit en accouchements au mois d'août et, plus encore, en juillet. Par rapport à un mois «normal», août enregistre un déficit en naissance d'environ $50 \%$ et juillet de près de $80 \%$. Un surplus de naissances est repérable en septembre - octobre, lui aussi redevable de la même explication. Le cumul demeure possible, les vacances d'été précédant le congé de maternité, d'autant qu'une partie du congé prénatal peut être reportée après la naissance de l'enfant.

Le mouvement saisonnier des femmes de l'enseignement est sans doute et de loin le plus typique parmi les différentes professions ${ }^{14}$, à la fois en raison du particularisme de cette profession (activité saisonnière) mais aussi par une forte communication entre enseignantes. Les salles des professeurs sont en effet le théâtre de discussions fréquentes à ce sujet, de conseils ou de compliments pour celles qui ont «bien programmé » leur enfant, créant une sorte d'émulation à la planification. «C'est vrai que dans le monde enseignant, on dit oh-là-là! on ne va pas perdre nos vacances ou... Ça, ça se dit beaucoup»; «D' ailleurs, à chaque fois que je dis que mon bébé est né au mois d'avril, mes collègues me disent que c'est bien calculé». Cette conduite est si répandue qu'elle peut être assimilée à un comportement «normé», lequel participe à évacuer d'éventuels sentiments de culpabilité liés au fait de programmer si finement la venue d'un enfant. «C'est même elle [amie infirmière scolaire] qui nous en a parlé avant que nous on puisse y songer. Le fait d'en parler, ça a dû quelque part me déculpabiliser de pouvoir penser un petit peu la même chose». Toutefois, si les avantages sont largement soulignés et relayés entre collègues, ce n'est pas du goût de tous, certains se refusant à programmer de la sorte. La venue d'un enfant se doit de rester désintéressée, aussi «naturelle» que possible.

\section{c) Des stratégies d'ordre professionnel}

Plus rares sont les femmes ayant planifié leur grossesse pour des raisons directement en lien avec leur activité. Les «artisanes, commerçantes, chefs d'entreprise» sont les plus enclines à prévoir la période d'accouchement pour ce motif ( $26 \%$ d'entre elles ont avancé une motivation professionnelle), juste devant les «cadres et professions intellectuelles supérieures » $(23 \%)$, les « ouvrières » ayant prêté un moindre intérêt à la question (9\%). Cependant, en isolant le mouvement saisonnier des naissances des différentes professions et catégories socioprofessionnelles, aucune tendance ne se dégage nettement. Les courbes se croisent, s'en-

14 D'après la nomenclature de l'INSEE en quarante-deux postes. Des professions particulières mais non isolées à ce niveau de classification laissent peut-être apparaître de fortes fluctuations saisonnières. Pour cette étude, nous ne disposons ni d'effectifs suffisants, ni d'un découpage des professions assez précis pour en faire état. 
tremêlent et se modifient d'une année à l'autre, ne laissant pas transparaître de normes comportementales propres à chaque PCS. L'interprétation demeure donc délicate. Quelques tendances peuvent néanmoins être dégagées.

Certains magazines destinés aux femmes «cadres d'entreprise» dispensent des conseils relativement à la «bonne période» de l'année pour avoir leurs enfants, de sorte que le congé de maternité empiète le moins possible sur l'activité. Ainsi, un accouchement au mois d'août permet de s'absenter à une période où l'activité économique est moindre, donc d'entraver le moins possible le bon fonctionnement de l'entreprise à laquelle on appartient ${ }^{15}$. Par ailleurs, le particularisme de certains emplois pour lesquels l'activité est saisonnière conduit aussi à une planification des naissances spécifique. Pour exemple, les femmes travaillant en cabinet d'expertise comptable cherchent à accoucher en dehors de la «période fiscale» (de janvier à avril), mois de l'année où l'activité est la plus importante. «Le plus gros de mon travail, c'est de janvier à avril. C'est un peu la folie et pendant cette période, mon chef n'apprécierait pas je crois que je sois absente pour ça». Les professions de la justice se caractérisent également par une activité variable selon différents moments de l'année, avec principalement moins de travail durant les vacances d'été. Ainsi, pour les avocates affiliées au régime des professions libérales, donc ne bénéficiant pas du congé de maternité au même titre que les salariées, il est intéressant de prévoir un accouchement à cette période. Le manque à gagner occasionné par une activité ralentie au moment de la fin de la grossesse et de l'accouchement est alors moindre. «La profession, c'était un élément important pour les questions de naître pendant les vacances parce qu'en fait, les tribunaux fonctionnent avec les vacances scolaires... Donc, ça permet de pouvoir s'arrêter très facilement en juillet et août, en tout cas de maintenir une activité réduite à cette période-là». Le cas des artisans-traiteurs offre une autre illustration. L'activité est en effet plus intense au moment des fêtes de fin d'année, ceci conduisant les femmes à éviter d'enfanter à cette période. «On s'était dit, on évitera le mois de décembre... c'est notre plus gros mois de l'année».

La planification peut également être fonction de la profession du conjoint. Pour la plupart des femmes, il n'est pas envisageable de concevoir un enfant sans l'aval de leur conjoint. Le projet doit être partagé par les deux parents et, pour de nombreuses femmes, il est important que le conjoint puisse être disponible au moment de la naissance de l'enfant. «On ne va pas programmer un enfant en plein mois de juillet-août, comme Etienne est agriculteur, on éviterait ce moment-là, parce que là à la limite, je pourrais peut-être aller accoucher toute seule. Si c'est le jour de la moisson, il ne sera pas disponible». Un creux des naissances se dessine ainsi aux mois de juillet-août chez les couples dont l'homme est agriculteur. Les stratégies uniquement déterminées par la profession du conjoint sont cependant peu fréquentes, de l'ordre de $3 \%$. La planification saisonnière représente essentiellement un enjeu pour les femmes.

15 Voir J. Fagnani, Un travail et des enfants. Petits arbitrages et grands dilemmes, Paris, Fayard, 2000. 


\section{d) Eviter d'accoucher en été}

Outre la santé de l'enfant à naître ( $c f$. supra), c'est aussi de celle de la mère dont il est parfois question. C'est en effet deux ou trois mois avant l'accouchement que les femmes ressentent les principales gênes occasionnées par leur état de grossesse. Ces désagréments sont généralement amplifiés lorsque la fin de grossesse survient au moment des jours les plus chauds de l'année. Certaines femmes prévoient en conséquence la période à laquelle elles seront enceintes. «C'est au niveau santé pour moi. Ce que j'ai fait attention, c' est que j' ai des problèmes de circulation sanguine, donc j'ai pensé à moi, pour ne pas passer un été avec ces problèmes de sang. Donc là, juin, je n'ai pas passé un été en étant enceinte. En fait, je voulais que ça tombe avant les jours chauds».

D'autres raisons s'ajoutent à celle-ci. Certains conjoints n'entendent pas renoncer à leurs vacances d'été, temps sacré de l'année à leurs yeux. Il convient alors de planifier la grossesse de sorte qu'elle ne vienne pas compromettre les projets de villégiature. Par ailleurs, accoucher au moment des congés d'été, c'est aussi risquer de ne pas pouvoir partager l' «heureux événement » avec ses proches, pour la plupart absents parce que partis en vacances. Ces raisons sont cependant rarement mobilisées pour justifier d'une planification en dehors de l'été.

\section{e) Souhaiter accoucher en fin d'année}

Accoucher en fin d'année présente également certains avantages, à la fois pour les conjoints et pour l'enfant. D'une part, une naissance survenant avant le premier janvier permet aux parents de déclarer une demie part supplémentaire (ou une part entière, suivant le rang de l'enfant) lors de leur déclaration d'imposition, ceci conduisant à des abattements non négligeables. Si l'enfant naît en décembre, il n'aura été présent dans la famille que quelques jours, n'aura donc engendré un «coût» que sur cette courte période mais sera comptabilisé comme ayant été présent sur l'ensemble de l'année écoulée. L'avantage est d'ailleurs compris par certains médecins qui acceptent d'utiliser les techniques médicales de déclenchement afin d'anticiper un accouchement de quelques jours pour permettre au couple de bénéficier de cet avantage: «décembre, généralement, est un bon mois. On sait à peu près l' expliquer, par rapport aux impôts... C' est vrai qu'on a beaucoup de femmes qui insistent pour être déclenchées en fin décembre» ${ }^{16}$. Ces considérations économiques ne sont le fait que de rares couples $(1,5 \%$ des femmes ayant planifié la période ont avancé cette unique raison).

D'autre part, en choisissant d'accoucher au dernier semestre, certaines femmes souhaitent que leur enfant puisse intégrer le système éducatif en avance, par rapport aux enfants nés en début d'année. Un enfant est en effet admissible au cours préparatoire (C. P.) dès lors qu'il a six ans révolus au moment de la rentrée scolaire (septembre) ou qu'il les aura avant la fin de l'année civile. Ainsi, un enfant né en décembre a, à l'entrée en C. P., pratiquement un an de moins qu'un enfant né en janvier de la même année. Certains parents souhaitent ainsi que leurs

16 Le déclenchement n'est cependant pratiqué que dans la mesure où les conditions médicales sont réunies (maturité du fœtus, col suffisamment ouvert notamment) afin d'éviter tout risque pour l'enfant et pour la maman. 
enfants soient parmi les plus jeunes, ceci leur autorisant un éventuel redoublement en cours de scolarité sans pour autant qu'ils aient de «retard».

\section{f) Des stratégies plus diffuses}

Quelques femmes enfin (6,6\% de celles ayant programmé la naissance), qui se recrutent principalement parmi les ouvrières et les inactives, ont prêté attention au signe astrologique de leur enfant. Ces stratégies poursuivent différentes finalités et se jouent à un niveau plus ou moins fin (souhait d'un signe en particulier ou stratégie d'évitement d'un signe). La répartition des naissances dans l'année est en conséquence plus diffuse et plus confuse, «en dents de scie». Le souhait de pouvoir choisir le signe astrologique peut ainsi traduire une projection que l'on se fait de l'enfant, de son devenir, des attentes ou des espoirs que l'on place en lui avant même sa naissance. «La première raison est peut-être surprenante, c'est des raisons de signes du zodiaque. Je voulais soit un Bélier, soit un Lion, soit un Scorpion (...). Je voulais avoir un enfant qui ait du caractère, une personnalité, de l'ambition et qui réussisse dans sa vie professionnelle et personnelle».

De ces quelques exemples présentés de manière non exhaustive ressort l'idée selon laquelle les stratégies sont multiples et variées. Les négociations présidant la planification d'une naissance font à la fois appel à des contraintes propres aux conjoints, à leur situation du moment, à l'originalité irréductible de chaque famille mais aussi à leur histoire personnelle, à la fois en tant qu'enfant et que parents.

\section{COMPRENDRE LA PLANIFICATION DES NAISSANCES À PARTIR DE LA NOTION D'«EXPÉRIENCE»}

Nous avons souhaité pousser l'investigation plus loin afin de mieux comprendre l'essence de certaines stratégies. Outre les diverses médiations ventant les avantages d'accoucher à une saison plutôt qu'à une autre (discussion entre amis, collègues, lecture dans des revues, etc.), la planification des grossesses peut être interprétée comme la conséquence d' «expériences» diverses. Deux principaux types sont retenus ici: «l'expérience du soi parent» qui correspondrait à l'expérimentation d'une première maternité (une première maternité à une saison donnée permet de mieux cerner les avantages et les inconvénients d'enfanter à cette période de l'année) et «l'expérience du soi enfant» qui serait à rapprocher de la manière dont une personne a vécu le fait d'être née un mois donné.

\section{a) «L'expérience du soi parent»}

Les femmes venant d'accoucher de leur deuxième enfant sont deux fois plus nombreuses que les primipares à déclarer avoir arrêté leur moyen de contraception «parce qu'elles se sentaient prêtes à avoir un enfant et qu'elles souhaitaient qu'il naisse à une certaine période de l'année» (19\% contre $8 \%$, respectivement). «L'expérience du soi parent» peut ainsi conduire un couple à ajuster ses comportements de fécondité de telle sorte qu'une seconde naissance survienne à une 
«meilleure» période de l'année que la première (expérience négative). Pour le Nord-Pas-de-Calais, sur la période 1991-2000, la répartition saisonnière des naissances de rang deux se distingue ainsi de celle des naissances de rang un par un surplus entre avril et juillet, soit à la «belle saison» (Insee, état civil). Les femmes ayant vécu la fin de leur première grossesse en été sont nombreuses à déclarer que «vivre la fin de sa grossesse au moment des fortes chaleurs d'été n'était pas facile à supporter» et tendent à avoir leur deuxième enfant à une autre période de l'année. De même, l'expérience d'une première maternité à l'automne ou en hiver incite davantage à programmer la naissance du deuxième enfant au printemps. «Elle, je l'ai eue en hiver et je ne suis jamais sortie pratiquement. J'ai voulu un printemps-été, c'est aussi à cause de l'expérience de la première». D'autres problèmes plus pratiques ont été soulevés, par exemple relativement à l'habillement. "L'hiver, je n'arrivais plus à mettre mes chaussettes, c'est pas évident quand on est enceinte. C'est aussi pour ça que je voulais un bébé d'été». La période de naissance du premier enfant peut aussi avoir eu des implications négatives au niveau professionnel pour la mère et l'inciter à prêter attention à la période d'accouchement des enfants suivants.

À l'inverse, la saison de naissance d'un premier enfant peut avoir été appréciée, qu'elle ait ou non été planifiée, incitant les parents à faire en sorte que les prochaines naissances surviennent au même moment de l'année (expérience positive). Parmi les couples qui viennent d'avoir un premier enfant, ceux dont l'accouchement a eu lieu au mois d'avril, de mai ou de juin sont plus nombreux que les autres à indiquer une préférence quant à la période de naissance de leur prochain enfant (35\% en moyenne contre $23 \%$ ) et ils envisagent davantage d'en programmer la venue au printemps (85\% contre 63\%). «Pour le premier, c'est par hasard, il est né au printemps, au mois de mai, et c'est vrai que Constance, on s'était dit c' est vraiment bien... On avait fait en sorte qu' elle naisse au printemps parce que avec Louis, on s'était dit c'est vraiment bien comme saison».

L'expérience d'une première conception permet par ailleurs de prendre conscience de l'existence d'un délai entre l'arrêt de la contraception et le début effectif de la grossesse et de l'intégrer à la programmation des naissances suivantes. «Vu le temps entre l'arrêt de la pilule et puis la conception de Louis [le premier], on avait prévu d' arrêter trois-quatre mois avant, de prévoir un temps et après, pour que ce soit au printemps». Ce «savoir d'expérience» permet ainsi d'essayer d'ajuster les «calculs » relatifs à la détermination de la période de naissance de ses enfants.

\section{b) «L'expérience du soi enfant»}

La relation envisagée entre le mois de la naissance d'un parent et celui de son enfant peut étonner. Mais, bien qu' a priori anecdotique, le mois de naissance des parents participe bel et bien à la programmation des naissances dans l'année. «L'expérience du soi enfant» ne joue cependant que de manière négative et répond principalement à deux causalités, chacune associée à une période définie de l'année: être né au moment des fêtes de fin d'année ou durant les grandes vacances. Dans le premier cas, les personnes interrogées ont gardé le souvenir d'un anniversaire passant « inaperçu » parce qu'à proximité de Noël, donc d'avoir été «lésées » en terme de nombre de cadeaux; dans l'autre cas, elles ont le souvenir 
de ne pas avoir pu célébrer l'événement avec leurs camarades de classe, la plupart étant partis en vacances.

Numériquement, «l'expérience du soi enfant» n'a qu'une incidence très limitée sur le mouvement saisonnier d'ensemble puisqu'elle ne s'adresse qu'aux conjoints nés à la charnière de deux années ou en juillet-août. Néanmoins, en ne considérant que les personnes nées à ces périodes particulières de l'année, l'influence n'est pas négligeable. En mettant en regard le mois de naissance des mères ayant déclaré qu'elles auraient préféré être nées à une autre période de l'année avec le mois de naissance de l'ensemble de leurs enfants (tous rangs confondus) lorsqu'ils ont été programmés dans l'année, une «corrélation» négative apparaît alors entre le mois de naissance de la mère et celui de son ou de ses enfant(s): un très net déficit en naissances se dessine d'octobre à janvier alors que la plupart des mères étaient nées à cette période. «Je suis née le 21 décembre, à côté de Noël, donc je ne voulais pas que mon enfant naisse à côté de Noël, parce qu'après, les grands-parents... quoi, on n'a plus d'anniversaire». De la même manière, on recense largement moins d'enfants nés en juillet ou en août. «Moi je suis du 15 juillet et c' est vrai que j' ai jamais pu fêter mon anniversaire avec mes copains, et même encore maintenant, parce qu' on partait toujours en vacances à ce momentlà et puis, de toutes façons y' aurait eu personne puisque tout le monde part en vacances. Alors c'était pas primordial, je m'en suis remis, ça va! mais quitte à pouvoir choisir, j' aimais mieux qu'on évite qu'il naisse en été».

Outre la date de naissance des conjoints, d'autres expériences inhérentes au passé des parents sont à la base de stratégies saisonnières mais elles restent marginales ${ }^{17}$.

\section{VUE D'ENSEMBLE ET PERSPECTIVES D'INTERPRÉTATION DU PROFIL SAISONNIER DE L'ENSEMBLE DES NAISSANCES EN FRANCE}

Le mouvement saisonnier des naissances reste dans son ensemble difficile à interpréter. Bien que la répartition des naissances «au fil des saisons » se reproduise presque à l'identique d'une année sur l'autre et qu'il semble pertinent de la lire comme la conséquence d'une planification de la part des couples (une femme sur quatre a cherché à programmer la naissance de son enfant dans l'année), les fluctuations restent d'une faible amplitude. Ceci ne laisse pas percevoir de manière franche l'existence d'un comportement normé. Toutefois, l'explication de la faiblesse des oscillations observées par le fait que les différentes stratégies se conjugueraient et se compenseraient entre elles ne paraît pas tenir. On constate en effet une suprématie des motivations qui alimentent les mois du printemps en naissances, les autres stratégies étant plus marginales. Toutes motivations confondues, la répartition des naissances programmées dans l'année reste très largement marquée par deux pics, l'un au printemps, l'autre au mois d'août, tendance tradui-

17 Pour illustration, lors d'un entretien, une maman expliquait qu'elle avait connu des difficultés durant sa scolarité (mauvais résultats, maladie), situation dont elle avait souffert durant son enfance. Ainsi, elle souhaitait offrir à ses enfants la chance d'avoir à la base un an d'avance à leur entrée au cours préparatoire (classe de C. P.) en faisant en sorte qu'ils naissent en fin d'année. 
sant la prééminence des stratégies liées à l'attrait de la belle saison et aux contraintes professionnelles. Par ailleurs, les représentations que se font les couples quant à la période de l'année jugée «idéale» pour avoir un enfant traduisent largement l'attrait suscité par la saison printanière. Près d'une femme sur deux déclare que s'il était possible de «choisir précisément le mois de naissance d'un enfant», elle le ferait. Se retrouvent largement en tête les motivations d'ordre climatique, $66 \%$ des femmes justifiant leur préférence par le fait que «ce serait plus agréable d'avoir un enfant à cette période de l'année », 23\% parce que «cela leur permettrait de cumuler leur congé de maternité avec leurs congés professionnels » et $20 \%$ car «pour la santé de leur enfant, il est préférable qu'il naisse à cette période», ces raisons pouvant se combiner entre elles ${ }^{18}$. Ces trois motivations conduisent globalement aux mêmes préférences quant au mois d'accouchement. A supposer «que l'on puisse choisir précisément le mois», $80 \%$ de ces femmes ont indiqué qu'elles choisiraient entre les mois d'avril, de mai ou de juin. La faible «visibilité» des stratégies au niveau global tient donc à d'autres facteurs, notamment au fait que peu de couples s'essayent réellement à planifier si finement leur fécondité.

Alors qu'une femme sur deux a émis une préférence quant à la période «idéale» pour accoucher dans l'hypothèse où il serait possible de choisir, on observe dans les faits que peu de femmes ont déclaré avoir cessé leur contraception un mois donné parce qu'elles souhaitaient que leur enfant «naisse à une certaine période de l'année» $(15 \%)^{19}$. Le concept «d'impensabilité » décrit par Philippe Ariès au sujet du contrôle des naissances dans la période antérieure au $\mathrm{XVIII}^{\mathrm{e}}$ siècle paraît se rejouer à ce fin niveau de maîtrise de la fécondité pour une fraction de la population. Certaines femmes ont en effet conscience que chaque rapport sexuel n'est pas nécessairement fécond. Elles ne motivent donc pas l'arrêt de leur contraception par le souhait d'avoir leur enfant un mois en particulier. Par ailleurs, les motivations des couples à programmer si finement la naissance de leur enfant ne paraissent pas, dans la plupart des cas, répondre à des impératifs forts mais sont davantage assimilables à des stratégies de confort. Les enjeux de la non réalisation du projet saisonnier initial sont généralement perçus comme minimes. Sans grande motivation, les chances de succès de la stratégie sont alors réduites.

Outre la proportion relativement faible de couples essayant de programmer la naissance de leurs enfants dans l'année, les éventuels échecs des stratégies mises en place amoindrissent encore l'effet de celles-ci sur le mouvement saisonnier d'ensemble. Si certains s'essayent à planifier, beaucoup n'y parviennent pas en raison des difficultés à anticiper le laps de temps séparant l'arrêt de la contraception et le début de la grossesse.

S'ajoutent à cela des stratégies visant à élire une période particulière pour concevoir. Dans la mesure où l'enfant est au yeux de ses parents l'expression de

18 Plusieurs réponses étaient possibles.

19 La formulation de la question était «Pour quelle raison avez-vous choisi d'arrêter toute méthode contraceptive à cette période-là? Pour des raisons de santé; Parce que vous sous sentiez prête à avoir un enfant et vous souhaitiez qu'il naisse à une certaine période de l'année; Parce que vous vous sentiez prête à avoir un enfant, peu importait la période de naissance de l'enfant dans l'année? Pour une autre raison». 
leur union, il n'est guère surprenant que certains souhaitent concevoir leur premier enfant au moment même où ils s'engagent à vivre ensemble, par le mariage, plus encore si l'union a été célébrée à l'Eglise. Ainsi, 16\% des couples mariés religieusement ont cessé leur moyen contraceptif au moment de leur noce, ce qui correspond de manière très manifeste à un moment plus propice que tout autre où les couples s'exposent au risque de conception. Si de nos jours le lien entre la saisonnalité des mariages et celles des premières naissances n'apparaît plus de manière flagrante ${ }^{20}$, l'hypothèse d'un lien entre les deux phénomènes ne peut donc pas pour autant être totalement rejetée.

Par ailleurs, certaines périodes de l'année paraissent plus favorables que d'autres pour que les couples se décident à concrétiser leur projet d'enfant. La période des vacances est ainsi sensiblement plus propice à la décision d'arrêter la contraception (juillet et août sont de loin les mois où les couples n'ayant pas planifié la naissance de leur enfant dans l'année ont cessé leur contraception).

D'autres phénomènes plus évènementiels favorisent des conceptions à des périodes précises de l'année. D'après nos données, le passage à l'an 2000, période charnière incluse dans les douze mois d'observation de l'enquête (novembre 1999-octobre 2000), a d'abord eu un effet sur certains comportements de fécondité. Environ $9 \%$ des femmes interrogées ont déclaré avoir tenu compte du passage à l'an 2000 dans la programmation de leur naissance. Plus généralement, on observe chaque année un pic des naissances en septembre et, plus spécifiquement, à la fin du mois, autour du $24^{21}$. Cette «pointe» de septembre proviendrait d'un supplément de conceptions autour du premier janvier, phénomène que Henri Leridon a poétiquement qualifié d' «étrennes de septembre »22. La Saint Sylvestre paraît ainsi être un moment propice aux rencontres et où «les couples déjà constitués peuvent trouver dans cette liesse et ces beuveries un aliment à leurs transports $»^{23}$. Dans une perspective comparable, il ne serait guère surprenant qu'un pic des naissances s'observe autour du 6 ou 7 novembre (selon que l'année est ou non bissextile), soit 266 jours après la Saint Valentin (le 14 février), fête des amoureux. Si la date est aussi favorable aux étreintes amoureuses qu'elle est faste pour les restaurateurs ou les fleuristes, novembre bénéficierait alors d'un «bonus » de naissances, bien utile pour ce mois traditionnellement le plus pauvre de l'année en accouchements. L'hypothèse d'une activité sexuelle plus intense de la part des couples le soir de la Saint-Valentin paraît effectivement confirmée, au regard de la répartition journalière des naissances dans le Nord-Pas-de-Calais pour l'année 2000 (davantage de naissances entre le 5 et le 9 novembre; Insee, état civil).

C'est ainsi un ensemble de facteurs qui contribuent, à des degrés différents, à la répartition des naissances « au fil des saisons ». Il n’est donc pas surprenant que

20 Voir par exemple Guy Desplanques et Michel De Saboulin, «Mariage et premier enfant: un lien qui se défait», Economie et Statistique, 1986, n. 187, pp. 31-45.

21 Ce maximum secondaire se dessine autour du 24 septembre, selon H. Leridon, «Les conceptions du premier janvier (ou: les étrennes de septembre)», Population, 1986, n. 3, pp. 599-602; entre le 25 et le 27 selon l'étude de J.-P. Sardon, «Amours d'été, enfants de printemps », Science et Vie, 1988, n. 163, pp. 139-145.

22 D'après le titre d'un article consacré à la question. Voir H. Leridon, «Les conceptions du premier janvier», art. cit.

23 Ph. Besnard, Mours et humeurs, p. 35. 
le profil saisonnier de l'ensemble des naissances soit peu accidenté et qu'en conséquence, il ne laisse guère entrevoir de comportements «normés».

Néanmoins, pris isolément, nous avons vu que certaines catégories de couples animées par des motivations fortes pour eux parvenaient à leurs fins dans d'importantes proportions (exemple des couples dont la femme est enseignante). La planification saisonnière implique d'abord que les couples aient un intérêt à choisir la période de naissance de leurs enfants dans l'année et qu'ils s'y essayent ensuite. Le maître mot est donc la motivation, associée à une fécondabilité sans soucis particulier. Les calculs effectués sont d'ailleurs parfois une affaire très sérieuse. "On savait que quand on faisait l' amour, on faisait un enfant... On avait calculé les dates donc on savait bien, on avait calculé les mois, on se doutait de quand il allait naître. Donc voilà, j'ai calculé mes jours, c'était de tel jour à tel jour, on l'a fait durant toute la semaine, régulièrement, professionnellement et puis pouf!». La récente mise à disposition de tests d'ovulation destinés à repérer les jours les plus fertiles du cycle, largement relayée par voie publicitaire (presse écrite $^{24}$, campagne d'affichage dans le métro parisien ${ }^{25}$, etc.), pourrait conduire une proportion plus importante de couples s'essayant à programmer la saison de naissance de leur enfant à y parvenir effectivement.

Institut national d'études démographiques (INED), Paris

arnaud.regnier-loilier@ined.fr

24 Voir par exemple l'article «Tests à domicile» publié dans la revue Fémina - La voix du Nord, 15 juin 2002.

25 Campagne d'affichage pour le test Clearplan dans le métro parisien en août 2002 avec comme slogan: «Vous pensez vraiment que je suis né dans un chou? Avec le test d'ovulation clearplan, identifiez les deux jours les plus fertiles de votre cycle pour que les spermatozoïdes ne finissent pas... dans les choux. Maximiser vos chances de concevoir votre bébé». Produit en vente en pharmacie. D'autres marques ont développé le même type de test. 